Contract No. and Disclaimer:

This manuscript has been authored by Savannah River Nuclear Solutions, LLC under Contract No. DE-AC09-08SR22470 with the U.S. Department of Energy. The United States Government retains and the publisher, by accepting this article for publication, acknowledges that the United States Government retains a non-exclusive, paid-up, irrevocable, worldwide license to publish or reproduce the published form of this work, or allow others to do so, for United States Government purposes. 


\title{
Influence of Film Structure and Light on Charge Trapping and Dissipation Dynamics in Spun-Cast Organic Thin-Film Transistors Measured by Scanning Kelvin Probe Microscopy
}

\author{
L.C. Teague ${ }^{1, a}$, M.A. Loth $^{2}$ and J. E. Anthony ${ }^{2}$ \\ ${ }^{1}$ Savannah River National Laboratory, Aiken, SC 29803, USA \\ ${ }^{2}$ Department of Chemistry, University of Kentucky, Lexington, KY 40506, USA
}

Herein, time-dependent scanning Kelvin probe microscopy of solution processed organic thin film transistors (OTFTs) reveals a correlation between film microstructure and OTFT device performance with the location of trapped charge within the device channel. The accumulation of the observed trapped charge is concurrent with the decrease in $\mathrm{I}_{\mathrm{SD}}$ during operation $\left(\mathrm{V}_{\mathrm{G}}=-40 \mathrm{~V}, \mathrm{~V}_{\mathrm{SD}}=-10 \mathrm{~V}\right)$. We discuss the charge trapping and dissipation dynamics as they relate to the film structure and show that application of light quickly dissipates the observed trapped charge.

Despite the numerous advances in organic-thin film materials and technologies over the last several years, challenges still exist for organic-based devices, ${ }^{1}$ including the ability to promote efficient charge transport and the short and long-term stability of device performance. Several groups have investigated the stability of organic-based devices over time-scales ranging from several minutes to several days or weeks. ${ }^{2-4}$ The majority of these studies focus on measuring shifts in the threshold voltage $\left(\mathrm{V}_{\mathrm{Th}}\right)$ as a function of time or gate bias $\left(\mathrm{V}_{\mathrm{G}}\right)$ stress. These shifts are attributed to a variety of factors including charge trapping at the semiconductor/dielectric interface, charge trapping within the organic layer itself (due to grain boundary structures), humidity effects, and chemical decomposition of the organic layer. Methods proposed to reduce these shifts include surface treatments, ${ }^{5,} 6$ addition of photoreactive interfacial layers, ${ }^{7,8}$ illumination, ${ }^{2,9,10}$ and environmental control. ${ }^{11-14}$

Proximal probe techniques, such as scanning Kelvin probe microscopy (SKPM), allow us to fully explore the structure- property relationships in working OTFT structures. ${ }^{14-}$ ${ }^{25}$ SKPM provides the ability to monitor changes in charge transport phenomena in both space and time, a capability not afforded by traditional electrical performance measurements

\footnotetext{
${ }^{a}$ Author to whom correspondence should be addressed. Electronic mail: lucile.teague@srnl.doe.gov
} 
alone. Previous studies by several groups utilizing SKPM have provided valuable insights into the performance of OTFT structures including: (1) contact-limited transport at the metal/semiconductor interface, ${ }^{15,18,26}$ (2) grain-boundary-limited transport; ${ }^{21}$ (3) charging of the organic material during gate bias stress; ${ }^{22}$ and (4) the presence of long-lived trapped charge in the device channel. ${ }^{23,27}$

Herein, we utilize SKPM to study the the time-dependent device performance and charge trapping phenomena of spun-cast OTFT structures which utilize difluoro 5,11bis(triethylsilylethynyl) anthradithiophene (diF-TESADT) as the active layer. We examine the relationship between the film structure and the trapped charge imaged by SKPM. We also discuss the time-dependent evolution of charge trapping and its subseqent dissipation.

DiF-TESADT was synthesized as previously reported. ${ }^{28}$ Spuncast OTFT devices were fabricated onto cleaned $\mathrm{SiO}_{2}$ substrates (n-type $\mathrm{Si}(100), 200 \mathrm{~nm}$ thermal oxide) with prepatterned, chemically modified Au source and drain electrodes using methods similar to those previously reported (see supporting information). ${ }^{21,22,29}$ Samples were mounted into a ceramic DIP, wire-bonded, and placed into a specially fabricated test-platform which allowed remote device operation and electrical testing (via Keithley SMU) while imaging. SKPM imaging of active devices was accomplished using the SKPM mode of a Park XE-70 AFM (Park Systems, Inc.). ${ }^{21}$ The time-dependent evolution of the trapped charge and its subsequent dissipation ${ }^{14}$ were imaged by scanning the same line in the device channel repeatedly ( $\sim 1 \mathrm{~Hz}$ scan rate) in SKPM mode. All devices were stored in a vaccum dessicator when not in use. A white light LED coupled into an optical fiber allowed full illumination of the OTFT (at an angle of $\sim 65^{\circ}$ with respect to normal, illumination power $<1 \mu \mathrm{W}$ ) during SKPM analysis.

The transport characteristics and measured mobilities for spun-cast devices studied herein are within the range of those previously reported, ${ }^{21,29,30}$ however, variations may be 
attributed to the specific deposition conditions (deposition solvent, deposition temperature length of contact treatment, and environment). An effective field-effect mobility of $\sim 0.086$ $\mathrm{cm}^{2} \mathrm{~V}^{-1} \mathrm{~s}^{-1}$ was calculated from the transfer characteristics of the OTFT device in Figure 1a ( $\mathrm{W}=2000 \mu \mathrm{m}, \mathrm{L} \approx 4 \mu \mathrm{m}$ ) in the saturation region and is typical of the devices studied herein (see Supporting Information).
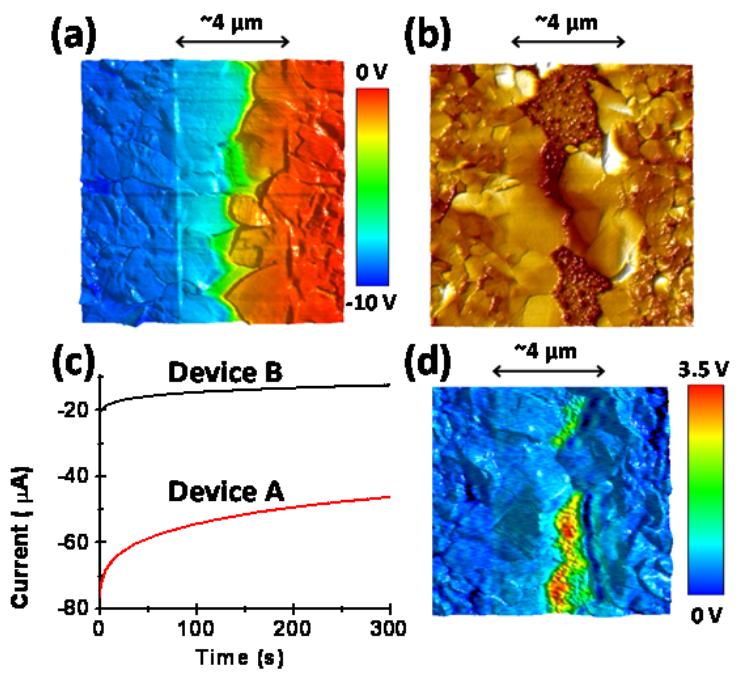

Figure 1. (a) SKPM image of a $10 \mu \mathrm{m} \times 10 \mu \mathrm{m}$ area of an OTFT device channel during operation $\left(\mathrm{V}_{\mathrm{G}}=-40 \mathrm{~V}, \mathrm{~V}_{\mathrm{SD}}=-10 \mathrm{~V}\right)$. The potential profile is overlayed on the topographic image. (b) topographic AFM image of a $10 \mu \mathrm{m} \times 10 \mu \mathrm{m}$ area of the device channel from a second OTFT device showing low-lying disordered regions (c) time evolution of $\mathrm{I}_{\mathrm{SD}}$ for devices show in $\mathrm{A}$ and $\mathrm{B}$ during operation $\left(\mathrm{V}_{\mathrm{G}}=-40 \mathrm{~V}, \mathrm{~V}_{\mathrm{SD}}=-10 \mathrm{~V}\right)$ (d) SKPM image of $\mathrm{a} \sim 8$ $\mu \mathrm{m} \times 8 \mu \mathrm{m}$ area of the channel of the device shown in Figure $1 \mathrm{~B} \sim 220 \mathrm{~s}$ after removal of a 5 minute bias stress $\left(\mathrm{V}_{\mathrm{G}}=-40 \mathrm{~V}, \mathrm{~V}_{\mathrm{SD}}=-10 \mathrm{~V}\right)$. The potential profile is overlayed on the topographic image and shows that positive charge is trapped in the low-lying disordered regions in the channel.

Figure 1(a) and (c) show the SKPM image of a $10 \mu \mathrm{m} \times 10 \mu \mathrm{m}$ area of the device channel and the corresponding time evolution of the source-drain current $\left(\mathrm{I}_{\mathrm{SD}}\right)$ during operation $\left(\mathrm{V}_{\mathrm{G}}=-40 \mathrm{~V}, \mathrm{~V}_{\mathrm{SD}}=-10 \mathrm{~V}\right)$. These results are consistent with previous studies of spun-cast diF-TESADT OTFTs in that grain-boundary limited charge transport is observed. ${ }^{21}$ However, in some devices, low-lying disordered regions similar to those seen in figure $1 \mathrm{~b}$ are observed. The presence of these regions suggests that the film growth mechanism in the device channel differs from that on the Au contacts, and is not surprising, given that it has 
been shown that chemical functionalization of the contacts can influence the overall film structure. $^{29,31-33}$

The corresponding time evolution of $\mathrm{I}_{\mathrm{SD}}$ during operation in this device (device $\mathrm{B}$ ) is shown in Figure 1c. Figure 1d shows an SKPM image of the device channel taken $\sim 220 \mathrm{~s}$ after the removal of bias stress (bias stress $\mathrm{V}_{\mathrm{G}}=-40 \mathrm{~V}, \mathrm{~V}_{\mathrm{SD}}=-10 \mathrm{~V}$ applied for 5 minutes). In the image, $\mathrm{V}_{\mathrm{G}}$ and $\mathrm{V}_{\mathrm{SD}}$ are grounded at $0 \mathrm{~V}$ and trapped positive charge is localized in these low-lying disordered regions. These results are consistent with reports of other OTFT materials by Marohn and co-workers ${ }^{23}$ in that localized regions of trapped charge are observed. We also note that the presence of trapped charge within the films is consistent with the observed decrease in $I_{D S}$ for the device over time during application of $V_{G}$ and $V_{S D}$ (Figure 2b). These results further confirm the relationship between the presence of trapped charge and device degradation.

From the time-dependent scanning Kelvin probe microscopy (td-SKPM) images (Figure 2, row 3) we can begin to understand more about the origins of the trapped charge, how it is distributed in the films, and how it is imaged via SKPM. Figure 2 highlights 3 different areas in the device channel (from 3 different devices made using the same procedure), each of which show slightly different relationships between film structure and trapped charge: (a) at a grain boundary, (b) in a low-lying disordered region, and (c) a crystalline region spanning across the entire channel.

In all cases, during application of a bias stress, positive charge is trapped within the device channel. This trapped charge and the dissipation of this trapped charge is observed in the td-SKPM images (Figure 2, row 3) immediately after the bias stress is removed. Line scans of all devices shown in Figure 2, row (d), do not indicate a contact potential difference at the contact edges, and suggest effective electronic coupling of the grains extending into the 
channel from the contacts. This suggests that the charge trapping and dissipation phenomena for these 3 areas are then strongly dependent on the structure of the organic layer.

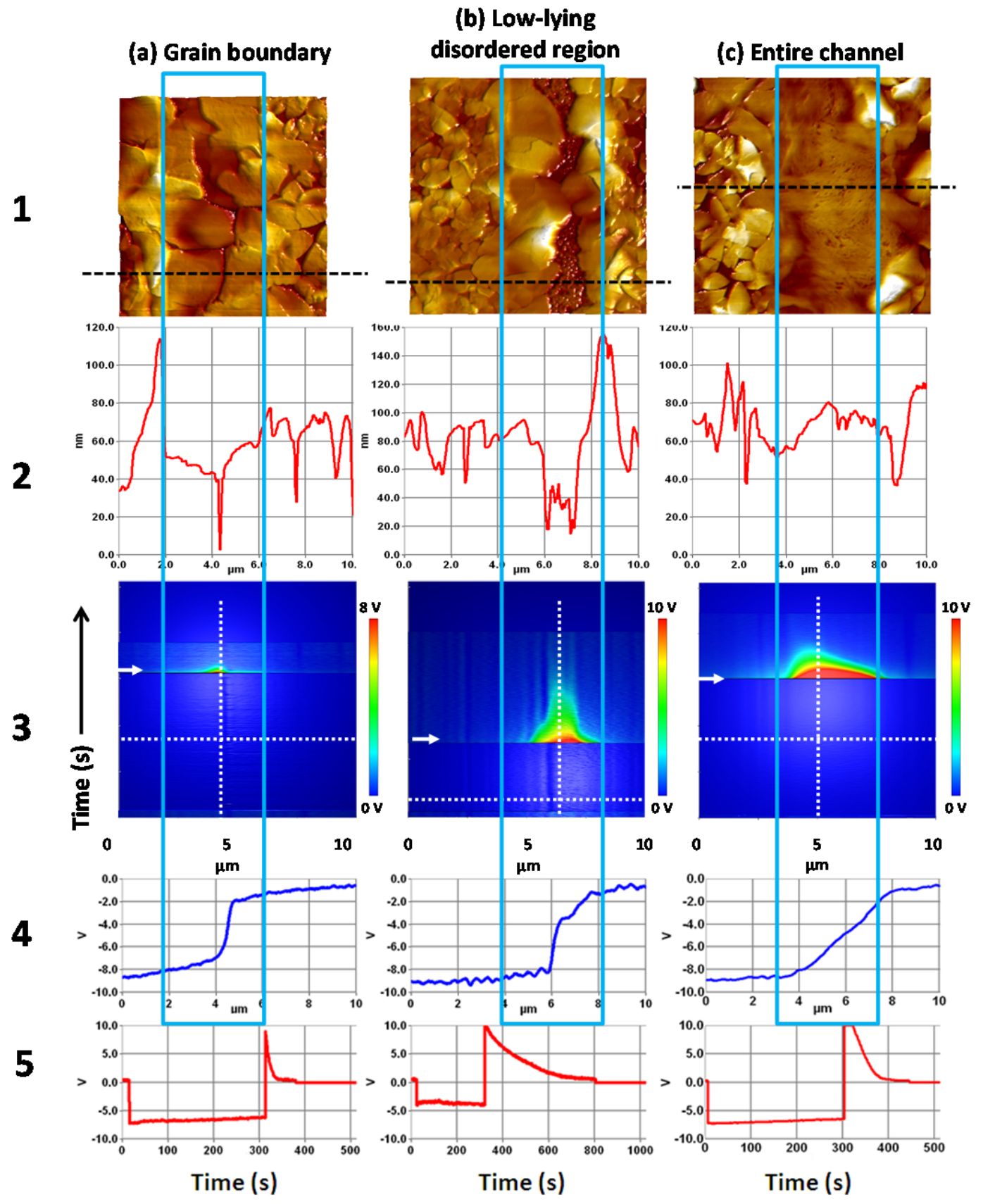

Figure 2. Columns (a-c) show $\sim 10 \mu \mathrm{m} \times 10 \mu \mathrm{m}$ topographic AFM images of 3 different types of structures in the device channel (row 1) and their corresponding charge trapping and dissipation characteristics as imaged by td-SKPM (images row 3, line profiles rows 4-5). Light blue boxes indicate the channel location in each plot and image. Dashed lines in row 1 images indicate location of topographic line profiles in row 2. White arrows in row 3 indicate the time at which bias stress $\left(V_{G}=-40 \mathrm{~V}, V_{\mathrm{SD}}=-10 \mathrm{~V}, 5 \mathrm{~min}\right)$ is turned off. Stored charge is 
observed when bias stress is removed. Dashed lines in row 3 images indicate locations of line profiles in the the $\mathrm{X}$ direction during application of bias stress (row 4) and in the Y direction over time (row 5) that show stored charge and subsequent dissipation. In each case, the amount, location, and subsequent dissipation rate of the trapped charge is dependent on the structure of the film within the channel as shown in the images and line profiles in rows 3-5. Drops in the potential across the channel, during the application of bias stress (row 4) coincide with the specific topographic features in the device channel.

In the case shown in column A, the trapped charge is observed by td-SKPM in regions where these well-coupled and well-ordered grains meet. When the device is "ON", a large drop in the potential profile in the channel occurs at this grain boundary (Figure 2, column A row 5 ). The majority of the stored charge dissipates within $\sim 60$ s once the device is turned "OFF". In the case shown in column B, the well-ordered grains extending from the contacts into the channel are well coupled to the electrodes, however, a sharp drop in potential is observed at the edge of these grains, when they meet a low-lying disordered region. The trapped charge in this low-lying disordered region takes $>5$ min to dissipate. Although the organic material is well-coupled to the contacts in the region shown in column $\mathrm{C}$, the film structure within the channel consists of much smaller grains, and is more disordered. In this case, both the hole and electron mobility are strongly affected by the structure, and the charge dissipation time is on the order of $2 \mathrm{~min}$. The slope of the voltage drop across the device channel (row 5) is inversely proportional to the hole mobility in the device. A rough comparison of this value for regions in Figure 2 (a) and (c) reveal that for both cases, the overall drop across the channel is $\sim 1.5 \mathrm{~V} / \mu \mathrm{m}(\mu \propto 0.66 \mu \mathrm{m} / \mathrm{V})$. However, if the large potential drop at the grain boundary in A is excluded, and only the voltage drop in the grains extending from the contacts is considered, the overall drop within these grains is $\sim 0.4-0.5 \mathrm{~V} / \mu \mathrm{m}(\mu \propto 2$ $2.5 \mu \mathrm{m} / \mathrm{V})$. This rough estimate also suggests that the faster dissipation rate of stored charge in $\mathrm{A}$ is due to more a well-ordered structure.

We have shown that the lifetime of the trapped charge is heavily influenced by the electronic coupling to the electrodes as well as the grain structure and order within the 
organic layer. The dissipation rate of the trapped positive charge is most likely due to effective injection and transport for free electrons in the film, which can recombine with the trapped positive charge. Thus, the overall presence and duration of trapped positive charge is related to the transport of free electrons in the films. Further support for this idea is found by examining the charge trapping and dissipation phenomena during illumination. Several groups have reported a light response of organic thin films. ${ }^{2,9,10,34-41}$ Here, we show via SKPM that when the OTFT is illuminated with white light during the application of bias stress, the amount of trapped charge is diminished (Figure 3(a), (c)). An associated increase in $\mathrm{I}_{\mathrm{SD}}$ is also observed when the OTFT is illuminated (150 s after device is turned "ON"). These combined results warrant further discussion with respect to the charge trapping and dissipation dynamics, which ultimately influence the device stability.

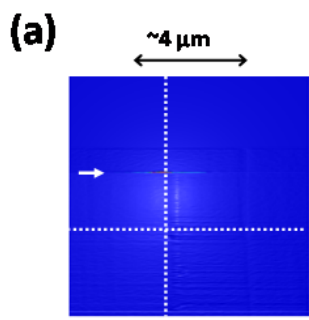

(c)

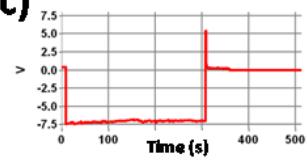

(b)

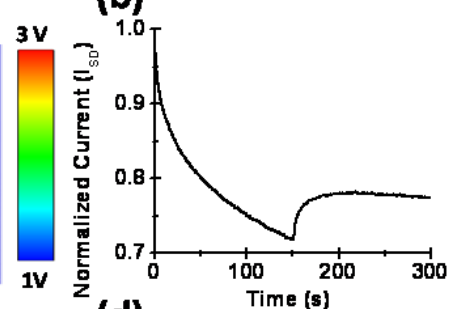

(d)

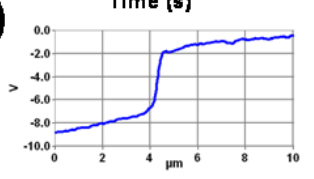

Figure 3. (a) td-SKPM image of transistor channel (same line/area as shown in Figure 2, column A). Dashed lines in (a) indicate the locations of line profiles show in (c) and (d) that show the stored charge and subsequent dissipation (c, Y direction) and potential drop during biasing ( $\mathrm{d}, \mathrm{X}$ direction). The white arrow in (a) indicates the time at which the 5 min bias stress $\left(\mathrm{V}_{\mathrm{G}}=-40 \mathrm{~V}, \mathrm{~V}_{\mathrm{SD}}=-10 \mathrm{~V}\right)$ was turned off. The OTFT was illuminated with a white light LED $\sim 150 \mathrm{~s}$ after the bias stress was turned on and remained on throughout the rest of the scan. (b) The time evolution of the source-drain current $\left(\mathrm{I}_{\mathrm{SD}}\right)$ for the device shown in (a) during operation shows an increase in $\mathrm{I}_{\mathrm{SD}}$ when the light is turned on. The amount of stored charge and subsequent dissipation observed following removal of the bias stress, are significantly different due to illumination, compared to Figure 2(a).

To explain this phenomena, we consider the following definition for the conductivity $(\sigma)$ of the semiconductor considering both holes and electrons: 
$\sigma=N_{e} e \mu_{e}+N_{h} e \mu_{h}$

where $N_{e}$ and $N_{h}$ equal the number of electrons and holes, respectively and $\mu_{e}$ and $\mu_{h}$ equal the electron and hole mobilities. For simplicity, we neglect the contributions of the distance over which the charges travel, although we note that, according to the Hecht relation, this will have some effect on the overall charge transport. ${ }^{42}$ When significant charge trapping occurs due to long and/or short term electron and/or hole traps, the overall conductivity is affected as is the case shown here in Figures 1-2. We consider for each trap, there exists a trapping rate $k_{t}$ and a detrapping rate, $k_{d}$. The detrapping rate for holes is a function of both the number of free electrons and the electron mobility, while the detrapping rate for electrons is a function of both the number of free holes and the hole mobility. The ratio of the detrapping rate to the trapping rate is then defined as the carrier lifetimes $\tau_{\mathrm{e}}$ and $\tau_{\mathrm{h}}$, such that:

$\tau_{h}=\left[\frac{k_{d h}\left(N_{e}, \mu_{e}\right)}{k_{t h}}\right]$ and $\tau_{e}=\left[\frac{k_{d e}\left(N_{h}, \mu_{h}\right)}{k_{t e}}\right]$

Accounting for the carrier lifetimes, we rewrite the conductivity equation as follows:

$\sigma=N_{e} e \mu_{e} \tau_{e}+N_{h} e \mu_{h} \tau_{h}$

In the case of diF-TESADT, we find from our SKPM studies that the hole trapping is much more significant than the electron trapping, such that the conductivity is affected most by changes in the hole mobility liftetime product, $\mu_{\mathrm{h}} \tau_{\mathrm{h}}$. For example, when the film is illuminated with high energy light (energies $\geq$ bandgap), a quantity of excitons is induced. Like free electrons in the system, these excitons can also dissociate and recombine with 
trapped holes, increasing the hole detrapping rate and resulting in free holes. ${ }^{9}$ These free holes may either be re-trapped or contribute to the overall conductivity of the system. Additionally, illumination at lower energies ( $\leq$ bandgap) can lead to excitation of trapped holes from mid-gap states into the transport band, thus increasing the conductivity of the system.

We note that herein, we illuminate over a range of wavelengths (energies) with a white light source which has sufficient energy to increase the current via both mechanisms. Therefore, in Figure 3, during illumination, the observed increase in conductivity is a result of an increase in the hole detrapping rate due to an increased number of free holes vs. trapped holes. The increase in free holes vs. trapped holes is a result of both induced photopairs and detrapping of holes from mid-gap states. Therefore, we observe a reduction in trapped charge in our SKPM images for devices operating in light vs. dark conditions. We expect that when $k_{d h}\left(N_{e}, \mu_{e}\right)=k_{t h}$, due to optimized illumination conditions, a steady state will be reached where the excess of free holes in the system contribute to the overall conductivity, such that the source-drain current can recover to its initial value, effectively stabilizing the device performance over time.

Herein, we have shown via td-SKPM measurements that the observed positive charge stored in the OTFT device channel and its subsequent dissipation rate is strongly dependent on the film structure and the electronic coupling to the source and drain electrodes. The results presented here suggest that the longer lifetime of charges trapped in the disordered regions of the organic material are due to insufficient pathways for free electron transport. These results are in agreement with previous studies of other polyacene OTFTs. ${ }^{23}$ Strong electronic coupling with the electrodes facilitates electron injection and well-ordered crystalline grains facilitate electron transport within the film to dissipate stored positive charge. We show that the amount of trapped positive charge can be reduced by injection of 
photons and photogenerated excitons from a white light source, resulting in a simultaneous increase in $\mathrm{I}_{\mathrm{SD}}$. We also discuss the dynamics of this recovery process. However, a more quantitative discussion of the observed charge trapping dynamics is difficult, because for each device, the overall trapping indicated by the decrease of $\mathrm{I}_{\mathrm{SD}}$ over time, is due to the convolution of the trapping and dissipation rates in various regions throughout the film. Within any given device, regions similar to those in Figure 2, (a-c) could be present, each of which contribute in some way to the overall trapping and dissipation rate.

LCT acknowledges financial support from the SRNL LDRD program. Cleanroom access was granted under CNM user proposal \#23377. Use of the Center for Nanoscale Materials was supported by the U. S. Department of Energy, Office of Science, Office of Basic Energy Sciences, under Contract No. DE-AC02-06CH11357. LCT thanks Dr. L. Ocola, Dr. D. Rosenmann, and Dr. R. Divan at CNM for their assistance with photolithography. LCT thanks SRNL colleagues Dr. R. Zidan, Dr. S.L. Garrison, and Dr. R. Lascola for their thoughtful discussions and K. Huffman, J. DeGange, M. Hudson, B. Blackmon, and J. Jones for engineering support. JEA thanks the Office of Naval Research (Contract \#N00014-11-10329) for their support of semiconductor development.

This manuscript has been authored by Savannah River Nuclear Solutions, LLC under Contract No. DE-AC09-08SR22470 with the U.S. Department of Energy. The United States Government retains and the publisher, by accepting this article for publication, acknowledges that the United States Government retains a non-exclusive, paid-up, irrevocable, worldwide license to publish or reproduce the published form of this work, or allow others to do so, for United States Government purposes.

1. S. S. Lee and Y. L. Loo, in Annual Review of Chemical and Biomolecular Engineering, Vol 1, edited by J. M. Prausnitz, M. F. Doherty and M. A. Segalman (Annual Reviews, Palo Alto, 2010), Vol. 1, pp. 59-78.

2. A. Salleo, F. Endicott and R. A. Street, Appl. Phys. Lett. 86 (26), 3 (2005).

3. S. G. J. Mathijssen, M. Colle, A. J. G. Mank, M. Kemerink, P. A. Bobbert and D. M. de Leeuw, Appl. Phys. Lett. 90 (19), 3 (2007).

4. S. G. J. Mathijssen, M. Kemerink, A. Sharma, M. Coelle, P. A. Bobbert, R. A. J. Janssen and D. M. de Leeuw, Adv. Mater. 20 (5), 975-+ (2008).

5. T. Miyadera, S. D. Wang, T. Minari, K. Tsukagoshi and Y. Aoyagi, Appl. Phys. Lett. 93 (3) (2008).

6. K. Lee, B. H. Lee, K. H. Lee, J. H. Park, M. M. Sung and S. Im, J. Mater. Chem. 20 (13), 2659-2663 (2010).

7. M. Marchl, M. Edler, A. Haase, A. Fian, G. Trimmel, T. Griesser, B. Stadlober and E. Zojer, Adv. Mater. 22 (47), 5361-+ (2010).

8. M. Marchl, A. W. Golubkov, M. Edler, T. Griesser, P. Pacher, A. Haase, B. Stadlober, M. R. Belegratis, G. Trimmel and E. Zojer, Appl. Phys. Lett. 96 (21) (2010).

9. A. Salleo and R. A. Street, J. Appl. Phys. 94 (1), 471-479 (2003).

10. L. W. Shang, Z. Y. Ji, H. Wang, Y. P. Chen, C. Y. Lu, X. Liu, M. X. Han and M. Liu, Ieee Transactions on Electron Devices 58 (7), 2127-2134 (2011). 
11. A. Sharma, S. G. J. Mathijssen, M. Kemerink, D. M. de Leeuw and P. A. Bobbert, Appl. Phys. Lett. 95 (25) (2009).

12. A. Sharma, S. G. J. Mathijssen, E. C. P. Smits, M. Kemerink, D. M. de Leeuw and P. A. Bobbert, Phys. Rev. B 82 (7) (2010).

13. A. Sharma, S. G. J. Mathijssen, T. Cramer, M. Kemerink, D. M. de Leeuw and P. A. Bobbert, Appl. Phys. Lett. 96 (10) (2010).

14. T. Hallam, C. M. Duffy, T. Minakata, M. Aando and H. Sirringhaus, Nanotechnology 20 (2), 8 (2009).

15. K. P. Puntambekar, P. V. Pesavento and C. D. Frisbie, Appl. Phys. Lett. 83 (26), 55395541 (2003).

16. J. A. Nichols, D. J. Gundlach and T. N. Jackson, Appl. Phys. Lett. 83 (12), 2366-2368 (2003).

17. L. Burgi, H. Sirringhaus and R. H. Friend, Appl. Phys. Lett. 80 (16), 2913-2915 (2002).

18. L. Burgi, T. J. Richards, R. H. Friend and H. Sirringhaus, J. Appl. Phys. 94 (9), 61296137 (2003).

19. L. W. Chen, R. Ludeke, X. D. Cui, A. G. Schrott, C. R. Kagan and L. E. Brus, J. Phys. Chem. B 109 (5), 1834-1838 (2005).

20. Y. Luo, F. Gustavo, J. Y. Henry, F. Mathevet, F. Lefloch, M. Sanquer, P. Rannou and B. Grevin, Adv. Mater. 19 (17), 2267 (2007).

21. L. C. Teague, B. H. Hamadani, O. D. Jurchescu, S. Subramanian, J. E. Anthony, T. N. Jackson, C. A. Richter, D. J. Gundlach and J. G. Kushmerick, Adv. Mater. 20 (23), 4513-4516 (2008).

22. L. C. Teague, O. D. Jurchescu, C. A. Richter, S. Subramanian, J. E. Anthony, T. N. Jackson, D. J. Gundlach and J. G. Kushmerick, Appl. Phys. Lett. 96, 203305 (2010).

23. M. J. Jaquith, J. E. Anthony and J. A. Marohn, J. Mater. Chem. 19, 6116-6123 (2009).

24. T. Hallam, M. Lee, N. Zhao, I. Nandhakumar, M. Kemerink, M. Heeney, I. McCulloch and H. Sirringhaus, Phys. Rev. Lett. 103 (25) (2009).

25. M. Tello, M. Chiesa, C. M. Duffy and H. Sirringhaus, Adv. Funct. Mater. 18 (24), 3907-3913 (2008).

26. D. J. Gundlach, L. Zhou, J. A. Nichols, T. N. Jackson, P. V. Necliudov and M. S. Shur, J. Appl. Phys. 100 (2), 13 (2006).

27. J. L. Luria, K. A. Schwarz, M. J. Jaquith, R. G. Hennig and J. A. Marohn, Adv. Mater. 23 (5), 624 (2011).

28. S. Subramanian, S. K. Park, S. R. Parkin, V. Podzorov, T. N. Jackson and J. E. Anthony, J. Am. Chem. Soc. 130 (9), 2706-+ (2008).

29. D. J. Gundlach, J. E. Royer, S. K. Park, S. Subramanian, O. D. Jurchescu, B. H. Hamadani, A. J. Moad, R. J. Kline, L. C. Teague, O. Kirillov, C. A. Richter, J. G. Kushmerick, L. J. Richter, S. R. Parkin, T. N. Jackson and J. E. Anthony, Nature Mat. 7 (3), 216-221 (2008).

30. S. K. Park, D. A. Mourey, S. Subramanian, J. E. Anthony and T. N. Jackson, Applied Physics Letters 93 (4) (2008).

31. D. J. Gundlach, L. L. Jia and T. N. Jackson, IEEE Electron Device Lett. 22 (12), 571573 (2001).

32. C. A. Di, Y. Liu, G. Yu and D. Zhu, Acc. Chem. Res. 42, 1573-1583 (2009).

33. C. A. Di, G. Yu, Y. Q. Liu, Y. L. Guo, W. P. Wu, D. C. Wei and D. B. Zhu, Phys. Chem. Chem. Phys. 10 (17), 2302-2307 (2008).

34. A. D. Platt, J. Day, S. Subramanian, J. E. Anthony and O. Ostroverkhova, J. Phys. Chem. C 113 (31), 14006-14014 (2009).

35. Y. Y. Noh, J. Ghim, S. J. Kang, K. J. Baeg, D. Y. Kim and K. Yase, J. Appl. Phys. 100 (9) (2006). 
36. Y. Y. Noh, D. Y. Kim and K. Yase, J. Appl. Phys. 98 (7) (2005).

37. C. H. Kim, M. H. Choi, S. H. Lee, J. Jang and S. Kirchmeyer, Appl. Phys. Lett. 96 (12) (2010).

38. C. H. Kim, S. H. Kim, S. H. Lee, S. H. Han, M. H. Choi, T. W. Jeon and J. Jang, Appl. Phys. Lett. 94 (8) (2009).

39. H. W. Zan and S. C. Kao, IEEE Electron Device Lett. 30 (7), 721-723 (2009).

40. M. Y. Cho, Y. D. Han, H. S. Kang, K. Kim, K. H. Kim, M. J. Cho, D. H. Choi and J. Joo, J. Appl. Phys. 107 (3) (2010).

41. M. Y. Cho, K. Kim, S. J. Kim, S. G. Jo, K. H. Kim, K. H. Jung, D. H. Choi, S. Kim and J. Joo, J. Appl. Phys. 108 (2) (2010).

42. G. F. Knoll, Radiation Detection and Measurement, Fourth ed. (John Wiley \& Sons, Inc., 2010).

\section{Supplemental Information}

The transport and transfer characteristics of a spun-cast diF-TESADT OTFT (W=2000 $\mu \mathrm{m}$, $\mathrm{L} \approx 4 \mu \mathrm{m})$ are shown in Fig. S1 and are typical of the devices studied herein. An effective field-effect mobility of $\sim 0.086 \mathrm{~cm}^{2} \mathrm{~V}^{-1} \mathrm{~s}^{-1}$ was calculated from the transfer characteristics of the OTFT device in the saturation region shown in Figure S1 according to the equation:

$\mu=\frac{2}{C_{i}} \frac{L}{W} \frac{\partial I_{D}^{1 / 2}}{\partial V_{G S}}$

where $\mu$ is the effective mobility, $C_{i}$ is the gate capacitance per unit area of the $\mathrm{SiO}_{2}$ thermal oxide layer, and $\mathrm{W}$ and $\mathrm{L}$ are the transistor width and length, respectively. The slope of the line of the linear part of $\sqrt{ }_{D}$ Vs. $V_{G S}$ was used for the value of $\frac{\partial I_{D}^{1 / 2}}{\partial V_{G S}}$.
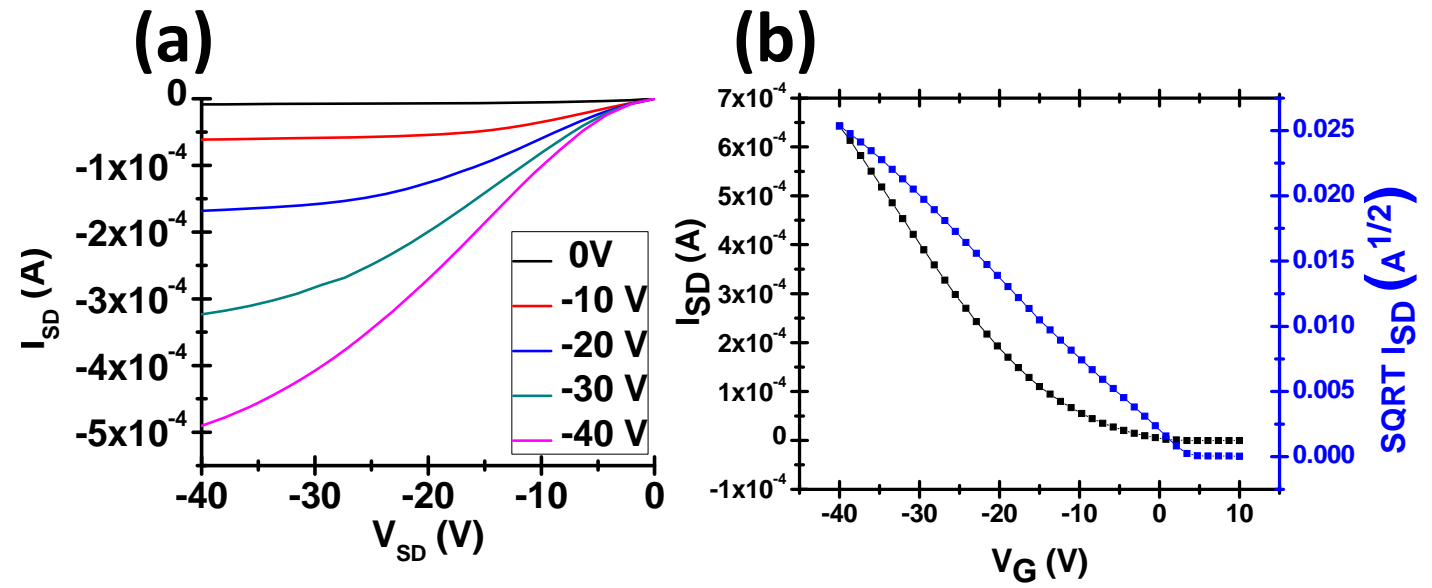

Figure S1. Transport (a) and transfer (b) characteristics (dark) of a spun-cast diF-TESADT OTFT $(\mathrm{W}=2000 \mu \mathrm{m}, \mathrm{L} \approx 4 \mu \mathrm{m})$ are shown. 
Standard photolithography and lift-off techniques were used to fabricate Ti/Au $(\sim 5$ $\mathrm{nm} / 45 \mathrm{~nm})$ source and drain contacts onto heavily doped n-type $\operatorname{Si}(100)$ wafers with a 200 nm thick thermal oxide layer. Patterned wafers were then coated with a thin photoresist layer prior to dicing and storage to protect the substrate surface during dicing and subsequent storage in laboratory ambient. Immediately before use, the protective photoresist layer was removed via sonication for $\sim 10$ minutes each in acetone and isopropanol, followed by a dI $\mathrm{H}_{2} 0$ rinse and $\mathrm{N}_{2}$ drying step. Samples were further cleaned via UV-ozone exposure for $\sim 10$ minutes, followed by a dI- $\mathrm{H}_{2} \mathrm{O}$ rinse and final $\mathrm{N}_{2}$ drying step. Chemical functionalization of the $\mathrm{Au}$ contacts on cleaned, patterned samples was accomplished via immersion for $\sim 30$ min in a $1 \mathrm{mM}$ solution of pentafluorbenzenethiol in ethanol followed by $10 \mathrm{~min}$ sonication in ethanol and $\mathrm{N}_{2}$ dry. The diF-TESADT active layers were then spuncast onto the cleaned and prepared substrates from a $\sim 1-2$ wt.\% solution in toluene (room temperature) at 2000-3000 rpm for 1 min. Samples were subsequently mounted into a ceramic DIP, wire-bonded, and placed into a specially fabricated test-platform for electrical and SKPM analysis. All devices were stored in a vaccum dessicator when not in use.

SKPM analysis of active devices was accomplished using the SKPM mode of a Park XE-70 AFM (Park Systems, Inc.) with an optical AFM head using conditions similar to those reported previously ${ }^{21}$.The specially fabricated test-platform containing the DIP structures used here allowed for electrical connections from a Keithley 2602A SMU to the sample source, drain, and gate contacts and enabled remote device operation while imaging in the AFM using SKPM mode.

Illumination studies were accomplished using a white light LED coupled into an optical fiber. The optical head of the AFM allowed the fiber to be positioned such that the OTFT could be fully illuminated (at an angle of $\sim 65^{\circ}$ with respect to normal) during SKPM 
analysis. The illumination power of the LED was measured using an optical power and energy meter (ThorLabs) and was $<1 \mu \mathrm{W}$ during SKPM analysis. 